\title{
Detection of partial discharge acoustic emission in power transformer
}

\author{
Yomna O. Shaker \\ Department of Electrical Engineering, Fayoum University, Egypt
}

\begin{tabular}{l} 
Article Info \\
\hline Article history: \\
Received Feb 26, 2019 \\
Revised Apr 24, 2019 \\
Accepted Jun 26, 2019 \\
\hline
\end{tabular}

\section{Keywords:}

Acoustic emission

Finite elements technique

Mathematical model

Partial discharge

\begin{abstract}
Partial discharge is one the most important factor that leads to deteroration and failure of the power transformer transformer. Acoustic emission detection is effective method to evaluate the health index of the power transformer using acoustic emission (AE) sensors for partial discharge (PD) measurement is considered as one of the most promising techniques to detect and localize PD activities inside the transformer tank. On the other hand, AE waves suffer from high attenuation and reflections while traveling from the $\mathrm{PD}$ source to the $\mathrm{AE}$ sensor. The modeling of the $\mathrm{AE}$ wave can help to understand the behavior of the AE PD signal during its travel. In this paper, the AE PD signal is assumed to be composed of different frequencies. This work aims to investigate the influence of the frequency value on the attenuation and arrival time of the acoustic wave.
\end{abstract}

Copyright () 2019 Institute of Advanced Engineering and Science. All rights reserved.

\section{Corresponding Author:}

Yomna Shaker,

Departement of Electrical Engineering,

Fayoum University,

Kiman Fared, Al Fayoum, Egypt.

Email: y.shaker@gmail.com

\section{INTRODUCTION}

Monitoring of PD is one of the tools to identify the health condition of transformer insulation system. When PD activities are initiated, the resulting energy is transformed into different forms as mechanical, electrical, and chemical energy. Among all PD detection techniques, there is a growing interest in the PD acoustic detection method because it overcomes several disadvantages of using electrical methods like low susceptibility to electromagnetic noise and cost effectiveness .

A significant effort has been exerted to understand the behavior of the PD acoustic wave while travelling from its source to the sensor. A mathematical model was introduced in [1] by solving the acoustic wave governing equations through finite elements technique. The presented model describes the propagation of PD acoustic wave in the whole medium (oil and steel) with contour plots to show the attenuation at different locations. Moreover, it examined the propagation properties under the effect of changing oil density as the temperature increased. The effect of frequency on acoustic PD attenuation was studied in [2] by applying pulses with different widths. As the frequency of PD pulse increases, this resulted in higher attenuation. This understanding of the PD behavior can be very helpful to predict the actual frequency ranges of the generated PD based on the received one. An approach in [3] presented the PD as a multiple of different sine waves with different frequencies and attempted to understand the interaction between them as it happens in PD pulse. This approach considers that all these sine waves will travel with the same velocity which is not accurate because each frequency will travel with different velocity as discussed by reference [4]. It has been mentioned that each frequency component of the PD wave will travel with different velocity and hence will arrive to the tank wall at different time which will lead to a higher degree of distortion of the received PD wave. 
The author consider two factors that will influence the acoustic wave attenuation and dispersion. The attenuation will be much greater for the high frequency component than the low frequency components. Also, the velocity of high frequency will be higher than the lower one which will result in more dispersion. These two effects will be combined together to fully understand the behavior of the acoustic PD wave [4].

From the previous discussion it is apparent that there are several factors that will influence the acoustic wave starting from the instant of occurrence till it reaches the transformer wall tank. These factors are: changing of velocity of the PD acoustic signal due to the frequency as well as the change in the attenuation of the different frequency components in the PD wave. In this paper, the propagation of acoustic wave in the transformer oil insulation system will be investigated considering those factors that influence its propagation by presenting a mathematical method that will be solved by finite elements technique to detect the frequencies of the wave accopmpined with their velocities.

\section{MATERIALS AND METHODS}

\subsection{Partial discharge modeling}

The PD signal can be considered as a point source of acoustic wave with a very short duration in the ultrasonic range from $20 \mathrm{kHz}$ to $1 \mathrm{MHz}[2,5]$. The exact shape of the pressure wave resulting from the PD is not known, but it is usually assumed to be a pulse as depicted in Figure $1[2,6]$.

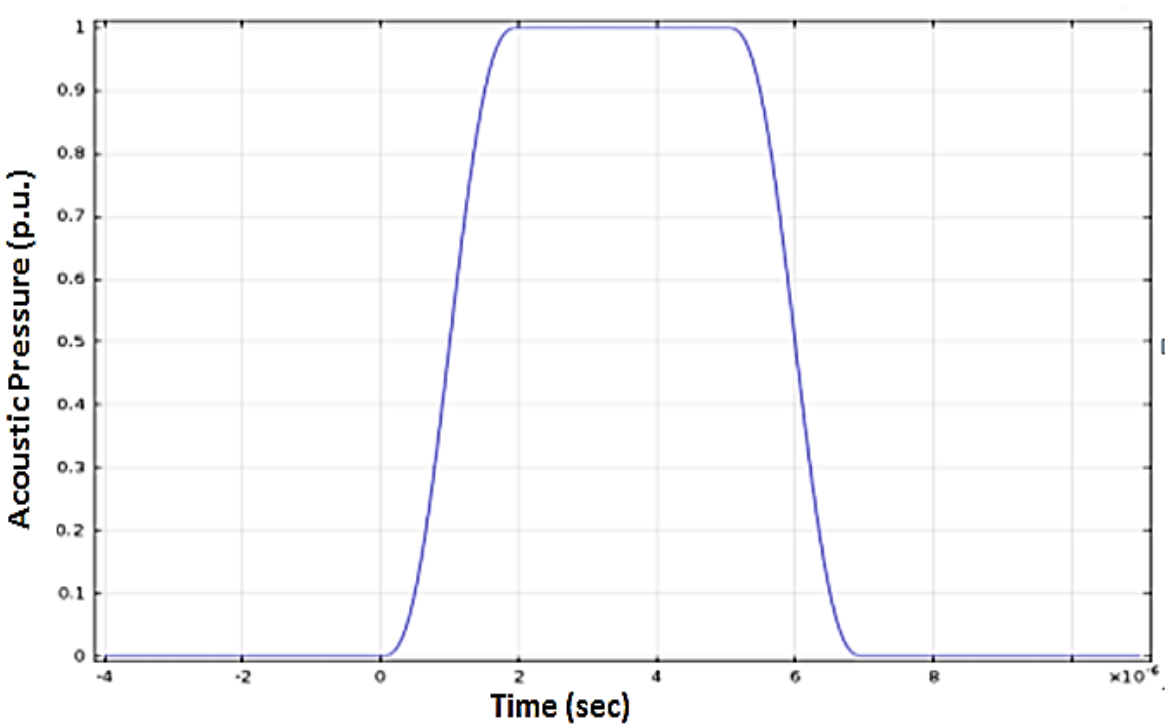

Figure 1. A typical waveform for the PD source

The acoustic wave propagation is evaluated by solving partial differential equations (PDE) using finite element technique. The standard partial differential equation that governs the propagation of an acoustic wave within 2-D homogeneous media is given by [5, 7]:

$$
\frac{\partial^{2} P}{\partial t^{2}}-C^{2}\left[\frac{\partial^{2} P}{\partial x^{2}}-\frac{\partial^{2} P}{\partial y^{2}}\right]=\delta\left(x-x_{o}\right) \delta\left(y-y_{o}\right) f(t)
$$

Where $\mathrm{P}$ is the pressure wave field in $\mathrm{Pa}$, $\mathrm{t}$ is the time in sec, $\mathrm{C}$ is the acoustic wave velocity in $\mathrm{ms}^{-1}$, and $\delta$ is the Dirac Delta function associated with the position of the PD source. In this paper, it is assumed that the steel tank dimension is $100 \mathrm{~cm} \times 100 \mathrm{~cm}$, the transformer oil density is $899 \mathrm{~kg} / \mathrm{m}^{3}$ and the PD source is assumed to be $25 \mathrm{~cm}$ away from the wall as shown in Figure 2.

The arrived acoustic wave at sensor (S1) location assuming the PD source, as shown in Figure 1, is depicted in Figure 3. The maximum peak value of the wave can be considered as a result of the direct oil path for the received wave and hence the arrival time of the peak value can be considered as an actual arrival time from the specified position of PD source. It can be understood from Figure 1 and Figure 3 that several changes happened to the wave during its propagation in the oil like reflection, attenuation and change of frequency. 


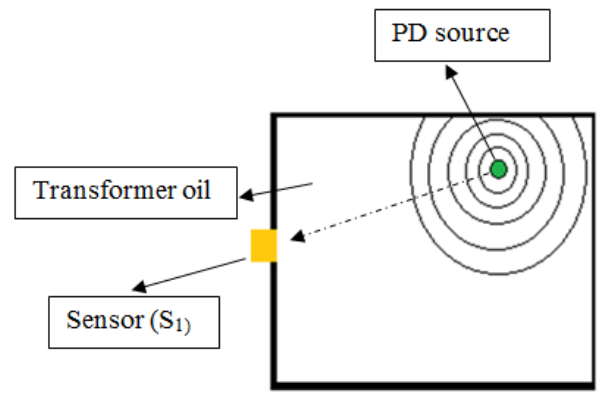

Figure 2. Schematic view for acoustic wave propagation

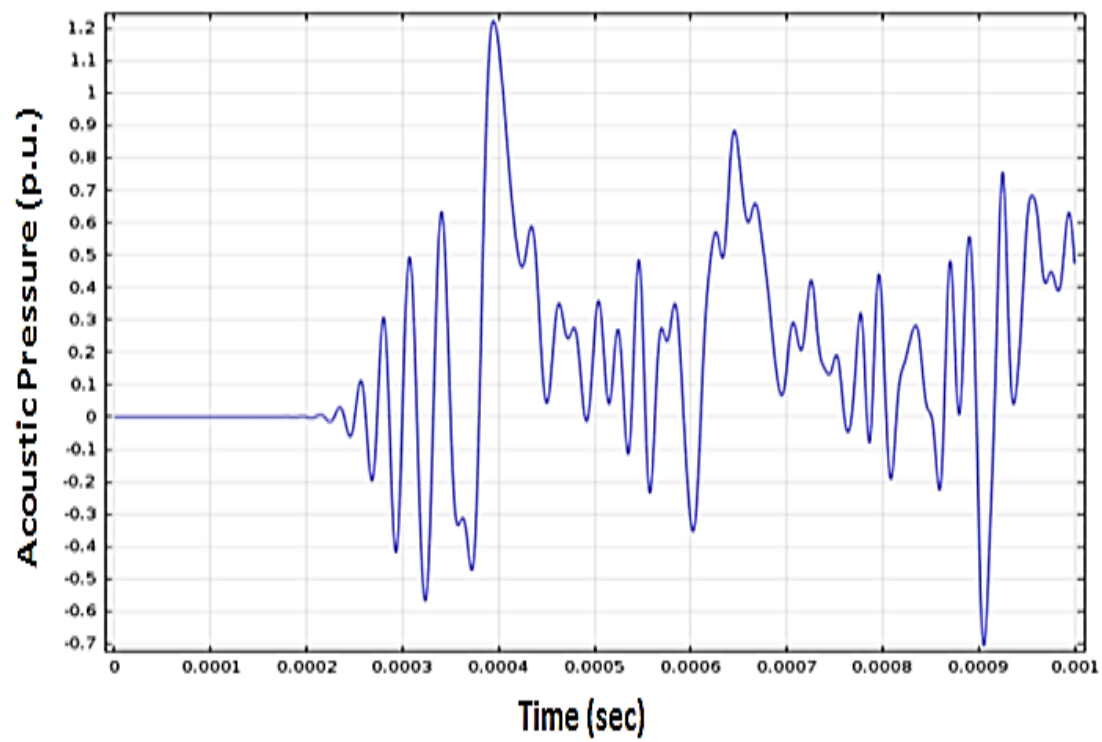

Figure 3. Typical received acoustic wave

\subsection{Frequency spectrum of partial discharge}

The measured PD is composed of different frequencies. A typical AE PD signal measured using the setup depicted in Figure 4 is shown in Figure 5 (a). Details about the setup can be found in [8]. The input wave consists of a group of frequency components which decays at different rates [4]. Figure 5 (b) shows the frequency spectrum of the received acoustic wave that ranges from 50-250 kHz. Instead of investigating the whole pulse, pure sine waves with $50 \mathrm{kHz}, 150 \mathrm{kHz}$ and $250 \mathrm{kHz}$ will be applied as a single source of acoustic wave to show how the system will respond to each individual frequency component.

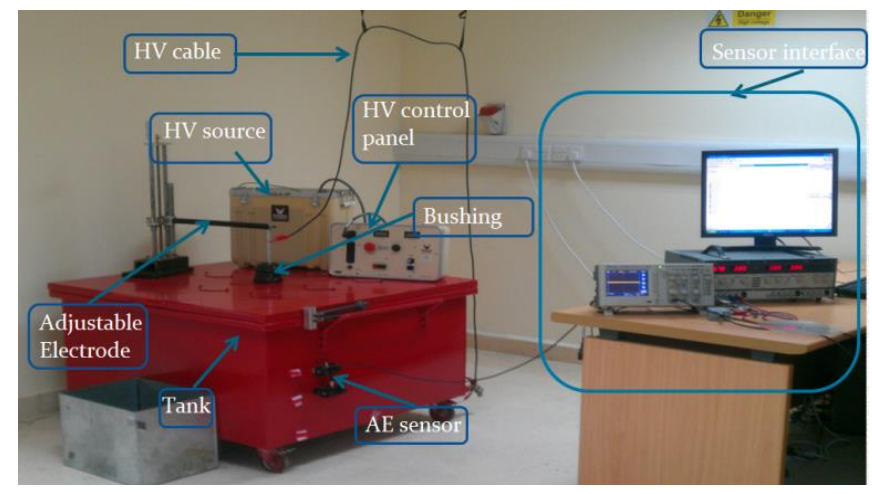

Figure 4. Overall system setup 


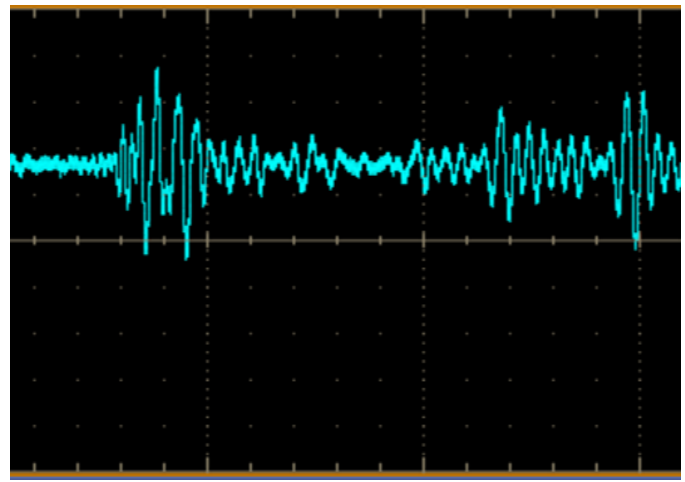

(a)

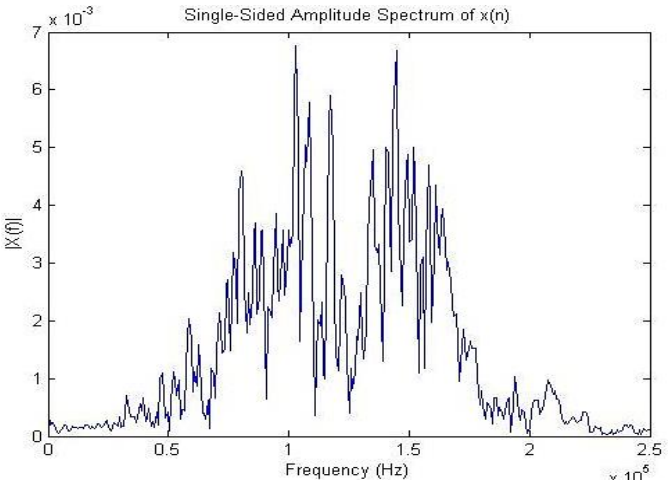

(b)

Figure 5. AE measured PD signal, (a) time domain signal measured at S1,

(b) frequency spectrum of the measured AE PD signal

\section{RESULTS AND ANALYSIS}

\subsection{Wave attenuation with individual frequency components}

As previously addressed, the input pressure was considered as a pulse that contains a lot of frequency components. Hence, to understand the behavior of the whole AE PD pulse, the behavior of individual frequencies $(50,150$ and $250 \mathrm{kHz})$ will be studied. The individual frequency components will be tracked at different distances from the PD source to get the value of their attenuation. It is very important to notice that the value of the sound velocity will be changed according to the frequency [4]. The velocity varies between $1000 \mathrm{~m} / \mathrm{s}$ for $50 \mathrm{kHz}$ and reaches $2700 \mathrm{~m} / \mathrm{s}$ for $250 \mathrm{kHz}$. This variation in velocity occurred as a result of the change in the acoustic impedance according to the frequency of the pressure source. Figure 6 shows the trace of a $50 \mathrm{kHz}$ frequency component in oil at the origin location, $5 \mathrm{~cm}$, and $15 \mathrm{~cm}$ from the source.

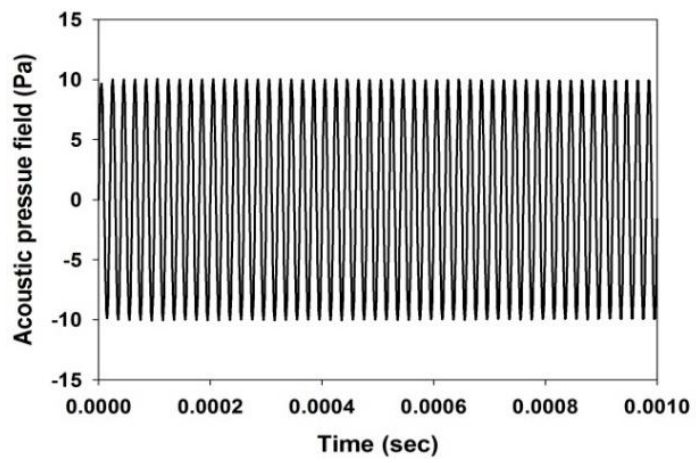

(a)

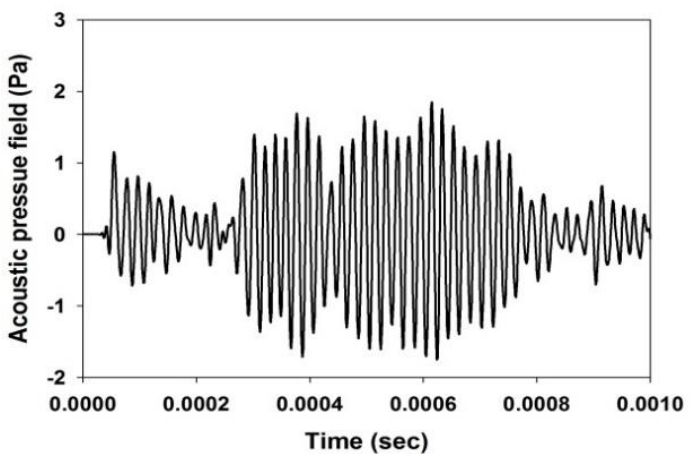

(b)



(c)

Figure 6. The trace of the $50 \mathrm{kHz}$ frequency component as a function of distance,

(a) The PD source (pure sine $50 \mathrm{kHz}$ wave), (b) $5 \mathrm{~cm}$ from source, (c) $15 \mathrm{~cm}$ from the source 
The wave is assumed to propagate with a speed of $1000 \mathrm{~m} / \mathrm{s}$ and the amplitude of the first peak of the wave decreased to about $6 \%$ at $15 \mathrm{~cm}$ from its original magnitude at the source. Figure 7 shows the relation between the peak value of the wave and the travelled distance from the source at different frequencies $(50,150,250 \mathrm{kHz})$. It is evident that the change in the wave attenuation due to frequency change is not significant at short distances from the source (up to $5 \mathrm{~cm}$ ). However, at longer distances, more attenuation is evident in the higher frequency components. Such change in the attenuation rate between the different frequency components will influence the final shape of the received wave.

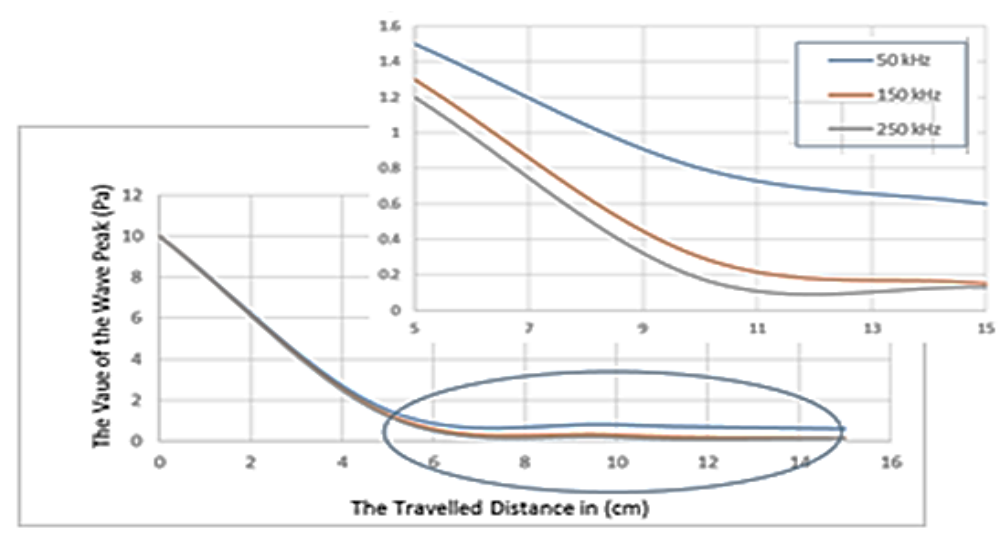

Figure 7. The value of attenuation at different frequencies $(50,150,250 \mathrm{kHz})$ with distance

\subsection{Effect of the different AE frequency components velocities on the PD localization}

In addition and as a result of the change in the velocity due to the variation of the frequency, the time arrival of each frequency component will be different. This means that the higher frequency components will reach the transformer tank wall before the low frequency components and this will result in further distortion in the shape of the received acoustic signal. To demonstrate the effect of frequency component speed on time arrival, a new input function will be investigated through the proposed model with a new location for the sensor (at $80 \mathrm{~cm}$ from the source). The input function as depicted in Figure 8 can be considered as a multiple of sine waves with different amplitudes and different frequencies as follows:

$$
30 \sin 50 \mathrm{kHz}+50 \sin 150 \mathrm{kHz}+20 \sin 250 \mathrm{kHz}
$$

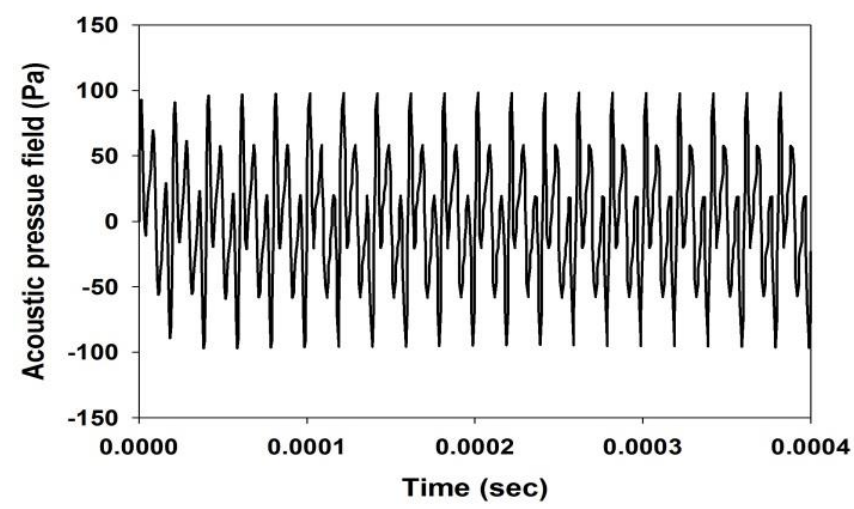

Figure 8. Input wave with multiple frequencies

It is important to study the effect of velocity changing on the arrival time as a result of frequency changing. First, it will be assumed that all frequencies will travel with a constant velocity of $1400 \mathrm{~m} / \mathrm{s}$. As a result and as shown in Figure 9, the arrival time for the peak value of the received AE PD signal is $584 \mu \mathrm{s}$. 
On the other hand, by using the sine waves separately with different velocities, the $20 \sin 250 \mathrm{kHz}$ component in (2) will travel with the highest velocity $(2700 \mathrm{~m} / \mathrm{s})$. This means that the highest frequency will arrive first compared to the other frequencies and the real arrival time of the input wave can be considered as the arrival time of the highest frequency component in this input. Figure 10 shows the received acoustic wave due to $250 \mathrm{kHz}$ component and its arrival time which is $297 \mu \mathrm{s}$ can be considered as the arrival time for the whole wave described by (2). Such change in the arrival time will result in a change in the calculated PD location and further deformation of the received AE PD signal.

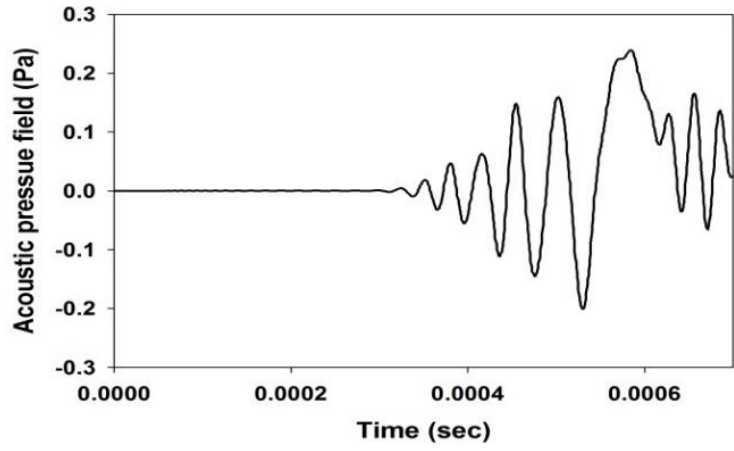

Figure 9. The received acoustic wave at $80 \mathrm{~cm}$ from the source with constant velocity



Figure 10. The received acoustic wave due to $250 \mathrm{kHz}$ component only

\section{CONCULSION}

The output acoustic wave received by the sensor can be analyzed using its frequency components. Each frequency component will travel with different velocity and hence reach the sensor with different arrival time. Moreover, the attenuation of each component increases with the value of frequency and travelled distance. By recollecting all frequency components, the distortion of the received acoustic wave could be completely understood and the location can be precisely detected.

\section{ACKNOWLEDGMENT}

This work was made possible by NPRP grant 5-044-2-016 from Qatar National Research Fund (a member of Qatar Foundation). The statements made herein are solely the responsibility of the authors.

\section{REFERENCES}

[1] S. A. Ashraf, et al., "3-D Simulation and Modeling of Acoustic Signals from Partial Discharge Activity", Annual Report Conference of Electrical Insulation and Dielectric Phenomena, 2009, pp. 619-622.

[2] P. Kundu, et al.," Simulation and Analysis of Acoustic Wave Propagating due to Partial Discharge Activity", Annual Report Conference of Electrical Insulation and Dielectric Phenomena, 2006, pp. 607- 610.

[3] D.Wotzka, T. Boczar,and P. Fracz, "Mathematical Model and NumericalAnalysis of AE Wave Generated by Partial Discharge," Acta Physica Polonica A, vol. 120, no. 4, pp.767-771.

[4] L. E. Lundgaard, "Partial discharge. XIV. Acoustic Partial Discharge Detection-Practical Application," IEEE Electrical Insulation Magazine, vol. 8, no. 5, pp. 34-42, Sep - Oct 1992.

[5] S. M. Markalous, S. Tenbohlen and K. Feser, "Detection and Location of Partial Discharges in Power Transformers using Acoustic and Electromagnetic Signals," IEEE Transactions on Dielectrics and Electrical Insulation, vol. 15, no. 6, pp. 1576-1583, 2008.

[6] M. Kozako, K. Yamada, A. Morita, S. Ohtsuka, M. Hikita, K. Kashine, I. Nakamura, H. Koide "Fundamental study on Partial Discharge Induced Acoustic Wave Propagation in Simulated Transformer Composite Insulation System," Proceedings of the 9th International Conference on Properties and Applications of Dielectric Materials, Harbin, China, Jul 2009.

[7] L. Tsang and D. Radar, "Numerical Evaluation of Transient Acoustic Waveform due to Point Source in a Fluid Filled Bore Hole," Geophysics, vol. 44, no. 10, pp. 1706-1720, Oct 1979.

[8] Mustafa Harbaji, Khaled Shaban and Ayman El-Hag, "Classification of Common Partial Discharge Types in OilPaper Insulation System Using Acoustic Signals," IEEE Transactions on Dielectrics and Electrical Insulation, vol. 22, no. 3, pp. 1674-1683, Jun 2015. 


\section{BIOGRAPHIES OF AUTHORS}



Yomna O. Shaker was born in Fayoum, Egypt, in 1976. She received the B.Sc. degree withHonors from Fayoum University in 1998 and the M.Sc. and Ph.D. degree from Cairo University, Cairo, Egypt, in 2003, and 2010 respectively. She is an assistant professor in the Electrical Engineering Department, Fayoum niversity, Egypt and a visiting scholar in American University in Sharjah. Her research andteaching interests are in high voltage equipment and Electrical Machines 